Best Practice Research in Clinical Gastroenterology

Cost effectiveness issues in gastrointestinal practice Volume 27, 2013 - special issue

\title{
Barrett's oesophagus screening and surveillance
}

Louisa G Gordon ${ }^{1 *}$ BEc, MPH, PhD, George C Mayne ${ }^{2}$ BSc(Hons), PhD

*Corresponding author

Affiliations:

1. Centre for Applied Health Economics, Griffith Health Institute, Griffith University, Logan Campus, University Dr, Meadowbrook, Queensland 4131, Australia louisa.gordon@griffith.edu.au, ph +61 733821320 fx +61 733821160

2. Flinders University, Department of Surgery, Flinders Medical Centre, Bedford Park, South Australia 5042, Australia

george.mayne@flinders.edu.au ph +61 882046088 fx +61 82046130 


\begin{abstract}
Endoscopic screening and surveillance of patients with Barrett's oesophagus to detect oesophageal cancer at earlier stages is contentious. As a consequence, their costeffectiveness is also debatable. Current health economic evidence shows mixed results for demonstrating their value mainly due to varied assumptions around progression rates to cancer, quality of life and treatment pathways. No randomised controlled trial exists to definitively support the efficacy of surveillance programs and one is unlikely to be undertaken. Contemporary treatment, cost and epidemiological data to contribute to costeffectiveness analyses are needed. Risk assessment to stratify patients at low- or high-risk of developing cancer should improve cost-effectiveness outcomes as higher gains will be seen for those at higher risk, and medical resource use will be avoided in those at lower risk. Rapidly changing technologies for imaging, biomarker testing and less-invasive endoscopic treatments also promise to lower health system costs and avoid adverse events in patients.
\end{abstract}




\section{Introduction}

Barrett's oesophagus is defined as a metaplastic change from normal oesophageal mucosa to columnar-lined epithelium containing goblet cells (1). It is commonly categorised into non-dysplasia, low-grade or high-grade dysplasia with the relative proportions in patients being $86 \%, 10 \%$ and $2 \%$ (2), respectively. Barrett's oesophagus arises in approximately $6-14 \%$ of patients who undergo endoscopy for symptomatic gastro-oesophageal reflux disease, but the condition goes underreported as many patients with Barrett's oesophagus are asymptomatic (3). Currently, patients with chronic gastro-oesophageal reflux disease are screened for Barrett's oesophagus, and as Barrett's oesophagus is associated with the development of oesophageal adenocarcinoma these patients are monitored under endoscopic surveillance. Oesophageal adenocarcinoma is an aggressive cancer with a poor 5-year survival rate (4), while its incidence has increased considerably in recent decades, particularly in Western populations. Observed risk factors for oesophageal adenocarcinoma include smoking and smoking duration; acid-reflux; obesity; alcohol; family history and diabetes, $(5,6)$ while a risk reduction is seen with frequent use of non-steroidal anti-inflammatory drugs (7). The strongest risk factor and only known precursor to oesophageal adenocarcinoma is Barrett's oesophagus. Patients with Barrett's oesophagus are at least 30 times more likely to develop oesophageal adenocarcinoma than patients without Barrett's oesophagus (8).

With 5-year survival rates for early stage oesophageal adenocarcinoma two to three times higher than late stage cases (from discussions with David and Tim $80 \%$ vs. $15 \%=5$ fold... from our not yet published data the Kaplan Meier shows no significant difference in overall survival between HGD/T1a-only pts and general Aust population), surveillance of patients with Barrett's oesophagus as a means to detect early stage oesophageal adenocarcinoma is recommended by several leading bodies $(9,10)$. As an early-detection cancer strategy, endoscopic surveillance of Barrett's oesophagus is controversial because the majority of patients undergoing surveillance do not develop oesophageal cancer and subsequently derive no benefit (11). The yield of early-stage cancers for patients within a Barrett's surveillance program varies widely from $1 / 285$ to $1 / 52$ patient-years,(1217) or $0.2 \%$ to $2 \%$ per year(4). However, advocates of surveillance suggest it is the only option for early detection, and since adenocarcinoma occurs through a known sequence of metaplasticdysplastic states, detecting pre-cancerous states within a surveillance program is critical to ensure optimal survival (1). While this appears to be a compelling argument in favour of routine surveillance of patients with Barrett's oesophagus, a number of complicating issues. A definitive diagnosis of dysplasia state can be difficult (18). Low grade dysplasia is difficult to differentiate from inflammatory reactive atypia. Furthermore, dysplastic lesions can be flat and difficult to detect with standard endoscopic techniques, and biopsy sampling protocols can miss focal lesions (Histopathology. 2007 Jun;50(7):920-7.; Am J Gastroenterol. 2000 Dec;95(12):3383-7; Hum Pathol. 2001 Apr;32(4):368-78). Surveillance methods are not always consistent (BMJ. 2006 Jun 3;332(7553):1320-3.) and the survival benefit conferred by surveillance is unclear due to the absence of randomized controlled trials on the efficacy of surveillance (18) + Gastroenterology. 2013 May 11. pii: S0016-5085(13)00715-4. doi: 10.1053/j.gastro.2013.05.004). Consequently, these issues continue to present a dilemma for clinicians and hospital administrators as to whether screening and surveillance endoscopies should be supported within routine programs.

There are also economic considerations for screening and surveillance of Barrett's oesophagus. Costeffectiveness analysis is the process of systematically comparing the relative health care costs and benefits of alternative strategies for the purpose of informing decision-makers of the strategy with the best value (19). Systematic assessment of incremental costs and effects requires knowledge of natural history and interaction with practice, and this is particularly relevant for oesophageal adenocarcinoma which has multiple strategies and pathways for monitoring, diagnosis and 
management, all conditional on the stage of disease at presentation (20). Although health provider costs may be of lesser concern to clinicians who will be focussing on optimizing outcomes for individual patients, ultimately, resource allocation decisions affect everyday clinical care in settings with budgetary pressures (21). It is mandatory for regulatory bodies in Canada, the UK, Australia and most of the Western world to evaluate cost-effectiveness of new technologies when considering potential government reimbursement.

Against this background, the present review introduces the economic issues of screening patients for Barrett's oesophagus and surveillance of those patients diagnosed with the condition. The current state of evidence is presented, along with the limitations of this evidence-base, and future directions on this topic. This review focuses particularly on surveillance strategies and assessments of their cost-effectiveness because this is where most of the research evidence is concentrated. Furthermore, screening cannot be implemented until surveillance strategies are shown to be cost effective.

\section{What are the economic issues?}

Cost-effectiveness studies for screening and surveillance of Barrett's oesophagus require robust data on the natural history of disease progression, the efficacy of treatments, and health resource use. However, data on all of these has been scarce, until more recently $(22,23)$. Oesophageal cancer suffers from the dual problems of being a relatively rare disease and having high resource use for adequate control (22). Research continues to be difficult as large numbers of patients are required in well-designed trials for evaluating the key outcome of 'detected cancer cases' arising from the progression of dysplasia in patients with Barrett's oeosphagus. Currently no such trial exists and it is unlikely that a large-scale multicentre randomised controlled trial of Barrett's surveillance or screening will be possible due to the low yield of cancer cases detected, long recruitment time and high participant numbers required. Consequently, this situation necessitates the use of economic modelling work to synthesize the 'best available' evidence and may use investigator assumptions to supplement information gaps.

It is also necessary to understand the prospective health resource use and outcomes for treating oesophageal adenocarcinoma or high grade dysplasia in a way that reflects real-world practice (24). An overview of the major practice patterns, natural history and management of oesophageal cancer and the associated health care resources used in a large Australian patient cohort $(n=1100)$ has been reported recently (24). However, the transferability of resource use to other nations is challenging due to clinicians adhering to different guidelines and associated practice patterns and also the organisational features of different health systems (22).

Treatment costs for oesophageal cancer are changing with newer technologies, and the associated impact on the economic benefit of surveillance programs is unknown. The high cost of the hospital episode for oesophagectomy is reported in several studies $(22,25)$. Surgical treatment is complex, and it is common for adverse events to occur, resulting in lengthy hospital stays and longer recovery periods in some patients. Previous work on patient-level resource data showed that that $24 \%$ of the patients undergoing oesophagectomy experienced at least one significant complication and the mean length of hospital stay for the surgical episode was 15.5 days (24). Many patients undergoing oesophagectomy were hospitalized for a longer period of time (24), whereas radiofrequency ablation and endoscopic mucosal resection are typically same-day procedures. However, these endoscopic procedures usually require several procedures per patient to complete a course of treatment, and ongoing intensive follow up surveillance (26). In assessing the subset of patients specifically with stage T1 adenocarcinoma or high-grade dysplasia, some economic efficiency might be gained from treatment pathways which involve less-invasive endoscopic therapies. This is driven 
by reduced treatment-related morbidity and mortality and lower treatment costs compared to surgery. This has clear implications for endoscopic and non-endoscopic surveillance based early detection strategies.

Few studies have assessed the cost-effectiveness of treatments for oesophageal cancer and highgrade dysplasia. Emerging strategies include substituting oesophagectomy with endoscopic therapies (radiofrequency ablation and endoscopic mucosal resection), or chemoradiotherapy, concentrating surgical work to high-volume centres, and early testing of tumour response during neoadjuvant chemotherapy by PET scan with subsequent targeted care. In a recent study (27), hypothetically increasing the proportion of patients with stage $\mathrm{T} 1$ adenocarcinoma who underwent endoscopic mucosal resection rather than oesophagectomy produced a smaller net benefit than for early detection strategies. Despite this, the results supported the use of endoscopic mucosal resection for $\mathrm{T} 1$ tumour treatment as shown by the positive net benefit, consistent with previous health economic reports of endoscopic techniques as preferred treatments for high-grade dysplasia and stage T1 adenocarcinoma versus oesophagectomy $(28,29)$. Pohl et al. 2009 assessed the costeffectiveness of combination radiofrequency ablation and endoscopic mucosal resection in patients with stage T1a oesophageal adenocarcinoma, and suggested that radiofrequency ablation was costeffective over a 5-year time frame (26). Similarly, a UK study by Boger et al. 2010 compared first-line radiofrequency ablation (with oesophagectomy for subsequent recurrence/progression) versus oesophagectomy in patients with high-grade dysplasia. Although modelling was limited to two years follow-up, they concluded that radiofrequency ablation was a cost-effective option. However, an Australian study found a high degree of uncertainty in the cost-effectiveness of radiofrequency ablation for low-grade dysplastic Barrett's oesophagus compared with surveillance (30).

\section{What economic methods have been used to evaluate screening and surveillance of Barrett's oesophagus?}

Several economic evaluations have been undertaken to provide information on the costeffectiveness of screening and surveillance of Barrett's oesophagus using standard health economic approaches. Full economic evaluations consider both the costs and the health effects of competing alternatives. Effectiveness in economic evaluation is often measured in quality-adjusted life years (QALYS), or life-years saved (LYS). A QALY is one year of life adjusted by a health state valuation (or utility), where death has a zero value and full health is valued at one. Several validated ? survey methods are available to elicit health state valuations from target populations.

Models in economic evaluation are generally used to extrapolate evidence from trials and other epidemiological data. A common modelling technique in health economic evaluation is the discrete time, single cohort Markov model. In such a model, a cohort of people of a given age is followed through successive health states, dependent on being in the intervention or comparator arm. The health states and the transitions between them determine both QALYs and costs, and these are aggregated to calculate an incremental cost-effectiveness ratio (ICER). The ICER is a ratio of the incremental costs to incremental effects of one treatment over a comparator and is interpreted as the additional cost per unit of outcome relative to the comparator. Willingness-to-pay thresholds are commonly used by health authorities as a guide of cost-effectiveness; when strategies are below the threshold they are judged as being cost-effective in that health system. The willingness-to-pay threshold in the US is often assumed to be $\$ 100,000$ per QALY while in the UK, the stated threshold is $£ 20,000-30,000$ per QALY. A strategy that improves outcomes while also saving costs compared to the next best option is considered 'dominant'.

Dealing with the uncertainty inherent in economic model estimates and their structure is a crucial aspect of economic evaluation (31). Using statistical distributions in place of point estimates 
provides a guide to the degree of variation possible in model parameters. A Monte-Carlo simulation can be used to re-sample from the probability distributions of all the variables (?) through many iterations to create a range of results with 95\% confidence intervals (31). Varying individual parameter estimates through a plausible range, known as one-way sensitivity analysis, and evaluating the impact on results, highlights key areas of uncertainty and indicates the degree of stability of the base results.

\section{Is screening for Barrett's oesophagus worthwhile?}

For a disease screening program to be acceptable, the World Health Organisation recommends certain criteria be satisfied (32). Some of these include: there should be a treatment for the condition, facilities for diagnosis and treatment should be available, the test should be acceptable to the population, the natural history of the disease should be adequately understood, there should be an agreed policy on whom to treat and the total cost of finding a case should be economically balanced in relation to medical expenditure as a whole. Information from research studies to inform these criteria are ongoing and further work is necessary in the case of screening for Barrett's oesophagus where these criteria are not strongly demonstrated.

Screening for Barrett's oesophagus precedes surveillance activity, however they have been previously combined in health economic studies (33), probably due to the involvement of endoscopy for both activities. In a 2009 review on the health economic evidence for screening (34), five studies were identified as meeting the inclusion criteria $(33,35-38)$. Studies were included if they examined conventional endoscopic screening followed by endoscopic surveillance of Barrett's oesophagus and used a cost-effectiveness or cost-utility framework. Other screening techniques included video capsule endoscopy and ultrathin unsedated nasal endoscopy. All studies targeted men with chronic gastro-oesophageal reflux disease.

All studies in the review used Markov health state transition models of hypothetical populations and observational data. Considerable variation in the estimates used in the models were apparent, for example, the prevalence of Barrett's oesophagus histological subtypes, progression rates, surveillance protocols, choice of costs and cost of oesophagectomy. All studies appeared to implicitly assume $100 \%$ compliance with the surveillance schedules. The estimated incremental costeffectiveness ratios varied between the studies:

- US\$10,440 per QALY for screening/surveillance of only persons with Barrett's oesophagus dysplasia(33),

- US\$11,254 per QALY for endoscopic screening/surveillance versus none (38),

- US\$55,764 per QALY for ultrathin nasal-endoscopic screening/surveillance versus none and US\$86,833 per QALY for endoscopic screening/surveillance (37),

- US\$12,140 per LYS for both metaplasia and dysplasia (36),

- US\$4,530 per LYS for screening/surveillance versus none and US\$24,850 per LYS for video capsule endoscopy (35).

The reviewers concluded that most studies in the review included optimistic assumptions that would have underestimated the incremental cost-effectiveness ratios. These included a lack of consideration for the possible negative impact on quality of life among individuals diagnosed with Barrett's oesophagus through screening, assumptions of $100 \%$ accuracy for endoscopic testing and diagnosis, no consideration of organizational arrangements to facilitate and maintain high uptake rates and allowing for different health care utilization of patients with reflux symptoms upon diagnosis of Barrett's oesophagus (e.g., more aggressive proton pump inhibitor medications or antireflux surgery) (34). In summary, the authors declared that there was insufficient evidence to 
recommend Barrett's oesophagus screening programs among patients with gastro-oesophageal reflux disease (34).

More recently, two additional studies have presented findings again using Markov model simulations for screening of Barrett's oesophagus $(39,40)$. Gupta et al. 2011 targeted a general population using endoscopy screening for Barrett's oesophagus at the same time as patients were already undergoing screening colonoscopy (40). Endoscopy screening only resulted in a cost per QALY gained ratio of $\$ 115,664$ while screening plus surveillance was $\$ 95,559$ per QALY gained. Benaglia et al. 2013 evaluated three options; endoscopy screening, cytosponge screening and no screening in a UK cohort of 50-year old men with a history of gastro-oesophageal reflux disease. They suggested that QALY gains were similar for the two strategies and therefore the comparisons were mostly concerning their costs. Treatment for men found to have Barrett's oesophagus with high-grade dysplasia or intramucosal cancer received radiofrequency ablation with or without endoscopic mucosal resection. Compared with no screening, the incremental cost per QALY results were US\$15,724 per QALY for cytosponge screening and US\$22,167 per QALY for endoscopy screening (39). This study advances previous work in that it concurs with latest natural history estimates of Barrett's oesophagus progression to cancer $(23,41,42)$.

\section{Is endoscopic surveillance of Barrett's oesophagus worthwhile?}

A recent systematic review assessed the evidence for endoscopic surveillance of Barrett's oesophagus (43). Seven studies met the inclusion criteria $(2,33,44-48)$ which involved a comparison of surveillance for individuals with non-dysplastic Barrett's oesophagus versus no surveillance, an outcome of either QALY or LYS and inclusion of both costs and benefits in the analysis. Figure 1 summarises the key results for the studies included in the review in terms of the incremental cost per QALY/LYS ratios of endoscopic surveillance versus no surveillance strategy. Two studies by Sonnnberg et al. reported incremental cost per LYS $(47,48)$.

Figure 1. Key results of incremental cost per QALY/LYS gained ratios for surveillance versus no surveillance 


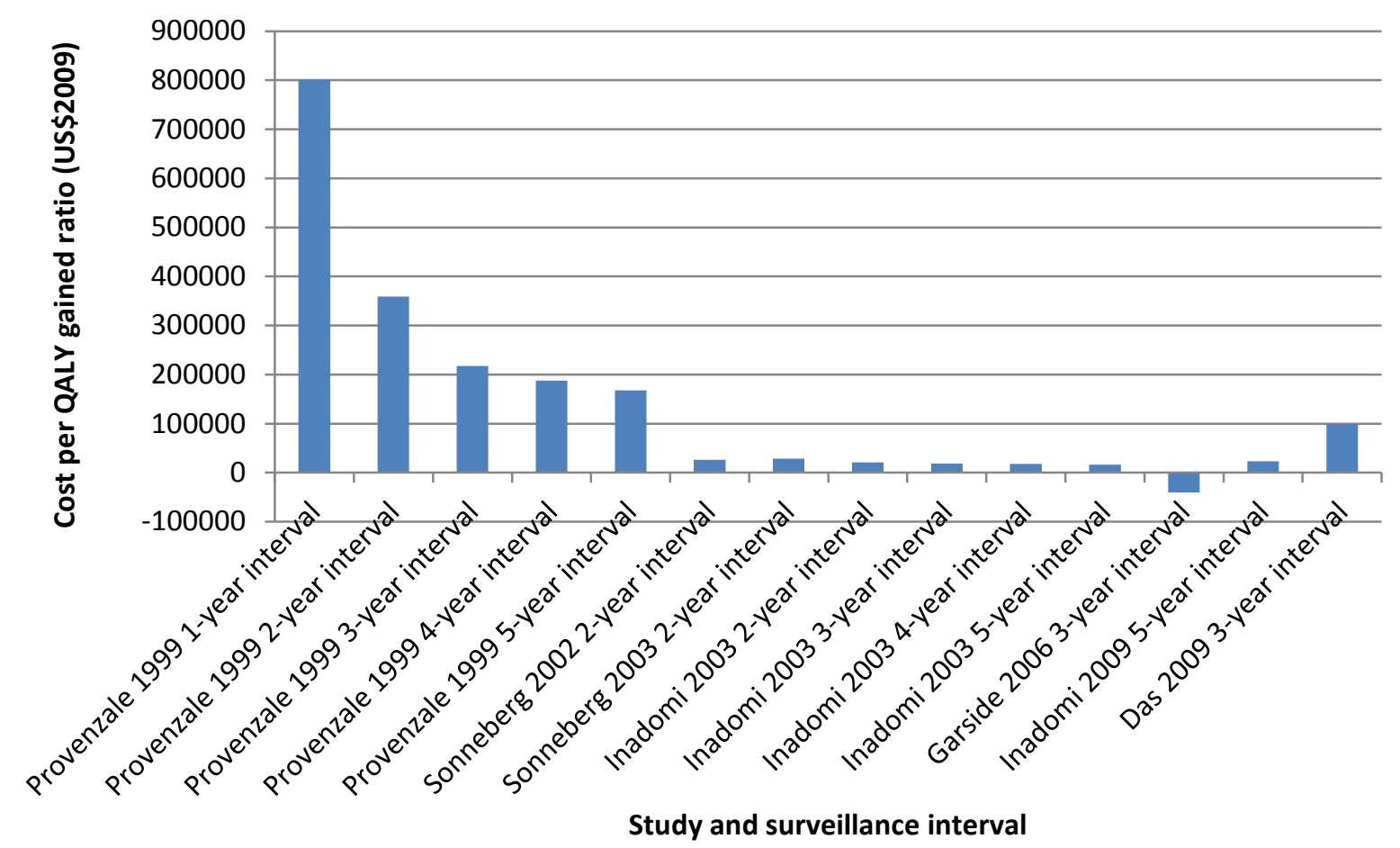

Source: Data from Hirst et al. (2011)(43)

Studies were published during 1999-2009, and with the exception of one UK study (2) the remaining studies were based on US populations. All studies employed decision-analytic Markov modelling with health state transitions to reflect disease progression. Modelled cohorts had starting ages ranging from 50 to 60 years and models were generally run for 25-30 years or until death (i.e., lifetime models). Rates of misdiagnosis and utility estimates often relied on author assumptions.

Figure 1 shows that the studies produced mixed results about the value of surveillance, ranging from surveillance being inferior (higher costs and lower outcomes) to no surveillance (2) to $\$ 801,041 \mathrm{per}$ QALY gained. With an acceptable willingness-to-pay threshold of up to $\$ 100,000$ per QALY gained in the US, many studies appeared to be cost-effective. Some studies suggest surveillance is not costeffective under any scenario tested $(44,46)$, while others concluded that surveillance is economically acceptable under certain conditions (for example, when the surveillance interval is lengthened (33, 45)).

The authors concluded that the studies in the review are now largely outdated as they were based on poor quality evidence (43). The evaluations were conducted according to best available evidence at the time of publication by recognized experts in the field of Barrett's oesophagus. Important inputs for modelling (e.g., quality of life, proportion of patients progressing among dysplasia grades, misdiagnosis rates) relied on assumptions by these experts. Mortality rates for oesophagectomy are also improving in specialist centres, and recent epidemiological studies have produced more rigorous estimates of the natural history of Barrett's oesophagus (23). Clinical practice has also improved with greater use of less invasive endoscopic techniques that promise to reduce treatment costs. Studies finding favourable cost-effectiveness ratios $(33,45,48)$ are likely to be due to a combination of the use of very high utility (quality of life) values ( 0.97) which seem counterintuitive, infrequent surveillance intervals ( 5 yearly) and less coverage of the costs involved (22). These studies also used higher rates of progression to cancer under surveillance $(\sim 0.50 \%)$ compared to more recent updates $(23,41)$. Moreover, analyses did not consider the clinical uncertainty of alternative management options for early stage cancer (43) such as endoscopic 
procedures $(28,43)$. In model-based economic evaluations, the results of uncertainty for all model inputs as well as considering results of structural uncertainty are recommended practice (49).

\section{Limitations of health economic evidence}

In both the health economic literature for screening and surveillance of Barrett's oesophagus, the main limitations for clear guidance in clinical practice are attributed to the following:

- There is no randomized controlled trial for screening and/or surveillance of Barrett's oesophagus to definitively demonstrate efficacy and therefore selection and other biases inherent in observational studies cannot be ruled out when assessing patient outcomes.

- Limited availability of model estimates: For example, economic models are consistently dependent on progression rates and quality-of-life estimates that in many economic evaluations, were largely reliant on author assumptions (43);

- Quality of analyses: Modelled studies have often not fully addressed all aspects of uncertainty in the analyses and later studies demonstrate more thorough attention to uncertainty;

- Transferability to other settings: The extent to which the findings are transferable to other settings will depend on several factors such as differences in practice patterns, health care prices and organisation of the health system. Although the costs used are specific for that country, the resource relativities to each other should be broadly similar across other Western countries.

- Program delivery: The assumptions made about the organization of the surveillance or screening programs may be overlooked. Such aspects as, the intensity of the co-ordination processes and follow-up procedures to ensure patient adherence to appointments and endoscopist adherence to standardized protocols (50) will differ across settings.

- Endoscopic screening/surveillance methods are not always consistent $(51,52)$, and a reproducible diagnosis of Barrett's oesophagus, with or without dysplasia, can also be difficult in individuals with short segments and hiatus hernia (53-55). Surveillance is likely to be less cost-effective if a lower compliance is modelled.

- Definition of Barrett's oesophagus: Finally, the UK-based British Society of Gastroenterology guidelines do not require patients to have confirmed intestinal metaplasia, and therefore, the results may not be comparable to patients undergoing surveillance in the US and other countries which define $\mathrm{BE}$ as requiring the presence of intestinal metaplasia.

\section{Future directions - Identifying high-risk individuals}

Targeting surveillance to high-risk individuals might maximize benefits and minimize unnecessary use of hospital resources. Intuitively, cost-effectiveness of selected populations for screening or surveillance should be achieved where QALYs gained are relatively high and their costs are potentially lower than strategies directed at 'any-risk' populations. However, at present the low specificity of molecular biomarkers in Barrett's oesophagus, which appears to be due to intertumour molecular heterogeneity, means that potentially low-risk patients would either need ongoing testing to capture false negatives, or we may need more or better markers. Molecular biomarker based risk stratification has therefore not yet entered clinical practice.

In the future, it is possible that predictive biomarkers might more accurately identify patients who are at risk of progressing to cancer, and this information could potentially be used to target these individuals for closer surveillance and to reduce surveillance for low risk patients, thus improving cost-effectiveness. Past work has shown a combination of critical abnormalities within the tumorsuppressor genes TP53 and CDKN2A, as well as DNA content abnormalities (tetraploidy and aneuploidy), are associated with a high risk of cancer progression (56), but this specific-biomarker 
approach appears to be hindered by inter-tumour heterogeneity. However, high throughput array based biomarker testing and/or deep sequencing have the potential to address the issue of heterogeneity, and are producing promising results (e.g. Hum Genomics. 2013 Mar 5;7:6. doi: 10.1186/14797364-7-6; J Pathol. 2013 Jul 8. doi: 10.1002/path.4212; Nat Genet. 2013 May;45(5):478-86. doi: 10.1038/ng.2591) that suggest that these technologies have the potential to deal with the level of molecular heterogeneity observed in Barrett's oesophagus (Biochem Soc Trans. 2010 Apr;38(2):374-9. doi: 10.1042/BST0380374; Cancer Prev Res (Phila). 2010 Nov;3(11):1388-97. doi: 10.1158/1940-6207; Curr Opin Gastroenterol. 2013 Jul;29(4):437-45. doi: 10.1097/MOG.0b013e328362282f), although at much greater expense that traditional single biomarker tests. It is therefore reasonable to envisage that biomarker testing of Barrett's oesophagus may become practical in the near future. While the precise components of future tests are not known at this stage, the likely costs and benefits can be estimated and included in models to predict their potential overall impact (57) .

Rubenstein JH et al. 2005 is the only published cost-effectiveness study involving a hypothetical biomarker testing option (58). The approach taken by Rubenstein JH et al. was to determine how sensitive and specific a biomarker test would need to be, and how cheap, to be cost-effective in surveillance. In more recent analyses, the cost-effectiveness of surveillance was markedly improved under the hypothetical scenario of biomarker testing based on data from the Seattle Barrett's Esophagus Research Program (PLoS Med. 2007 Feb;4(2):e67), with acceptable limits of sensitivity and specificity, which differentiated surveillance program participants into low- and high-risk groups, and subsequently with more targeted treatment pathways and higher proportions of pre- or early-stage cancers detected in the high risk group (Gordon et al. 2013, unpublished). The cost-effectiveness of an alternative biomarker strategy remained favourable if patients testing negative for biomarkers did not receive surveillance in the first five years and received 2-yearly surveillance thereafter. In addition, if endoscopy surveillance of patients with non-dysplastic Barrett's oesophagus was scheduled less frequently, either 3- or 5-yearly and/or annually for low-grade dysplasia, the costeffectiveness of surveillance would be considered acceptable in most health systems. This, however, assumes that no cancers progress to advanced stage disease under such modified surveillance protocols, and there is only limited evidence available to support this (59).

A significant advantage of biomarker testing is the capability for objective assessment of risk, and it could therefore replace risk assessment based on the identification of low-grade dysplasia, which has the problem of variable histopathological interpretation, and the problem of lesions being missed by biopsy sampling. In our managed surveillance program, which has a high level of compliance to surveillance intervals and biopsy sampling (BMJ. 2006 Jun 3;332(7553):1320-3), 50\% of patients that developed high grade dysplasia or adenocarcinoma under surveillance progressed without detection of low grade dysplasia (Gordon et al. 2013 unpublished). However, no studies to date have reported the efficacy of longitudinal biomarker testing to determine whether the false negatives from initial testing can be detected during follow up. Therefore, the appropriateness of biomarker testing, its efficacy within a surveillance program, its feasibility and its acceptance are yet to be determined.

Further research involving patients with positive biomarkers and additional high-risk clinical factors such as being male, the presence of oesophagitis, length of Barrett's oesophagus, and length of time with Barrett's oesophagus (41) is warranted on economic and efficacy grounds to elicit outcomes from a more targeted high-risk surveillance population. Risk assessment procedures that propose the combined use of molecular, clinical and epidemiological markers are needed and require evaluation of their effectiveness and cost-effectiveness.

\section{Other impacts on surveillance}

Despite encouraging initial results, the treatment of low-grade dysplasia with radiofrequency 
ablation is still an area of controversy (30), and there is no consensus that these patients should undergo radiofrequency ablation. There is also a lack of outcome data for radiofrequency ablation for low-grade dysplasia. It is certain that the costs of the surveillance would be higher due to the cost of radiofrequency ablation treatment added to ongoing endoscopic surveillance (30). It is clear that cost-effectiveness for radiofrequency ablation for low-grade dysplasia can only be achieved if patients undergoing ablation can be discharged from ongoing follow-up, or have sufficiently reduced surveillance, and there is currently no evidence to support these strategies.

There are various treatment options for Barrett's oesophagus which overlap with those for treating symptomatic gastro-oesophageal reflux disease, namely proton pump inhibitor medications and anti-reflux surgery. The goal of these treatments has been to treat reflux symptoms, with a hope that this might to limit progression to cancer. However, neither anti-reflux therapy produces predictable regression, or prevents cancer development $(60,61)$. Patients receiving these treatments will need to be identified in upcoming research on the clinical utility of surveillance activity as they are likely to confound surveillance outcomes and cost-effectiveness analyses.

\section{Summary}

With increasing pressures on health systems to contain health care costs, information on the costeffectiveness of existing and proposed strategies is increasingly contributing to decision-making. Economic evaluations are designed to assist with efficiently allocating scarce health care resources, that is, to minimise costs for given health outputs. The cost-effectiveness of appropriate management strategies for patients with Barrett's oesophagus must be considered. Using current estimates of the malignant potential of Barrett's oesophagus in the wider population versus those reported in surveillance program audits, surveillance of all patients with non-dysplastic Barrett's oesophagus may not be cost-effective. However, further work to identify high-risk individuals, perhaps in the future using a biomarker based strategy, might enable endoscopy surveillance to be tailored to high-risk individuals, and thereby improve the economic acceptability of endoscopybased surveillance of Barrett's oesophagus.

\section{Practice points}

- No randomised clinical trial exists to confirm the efficacy of surveillance of Barrett's oesophagus

- Traditional endoscopic surveillance of patients with non-dysplastic Barrett's oesophagus may not be cost-effective and remains controversial until updated cost-effectiveness analyses prove otherwise

- Using Clinical Practice Guidelines and consensus statements to guide practice around surveillance protocols will increase the cross-comparison of research audits and could be used to feed into cost-effectiveness analyses.

\section{Research agenda}

- Emerging technologies used in the pathways of care for patients identified for screening and surveillance of Barrett's oesophagus need to be assessed for their cost-effectiveness

- Identification of high-risk individuals via biomarkers or other known risk-factors shows promise in improving the cost-effectiveness of endoscopic surveillance and research evidence to confirm this is required.

\section{Funding sources}

L Gordon was supported by a National Health and Medical Research Council Early Career Fellowship \#496714. 
Conflict of interest:

The authors' have none to declare. 


\section{References}

1. Shaheen NJ, Richter JE. Barrett's oesophagus. Lancet. 2009;373(9666):850-61.

2. Garside R, Pitt M, Somerville M, Stein K, Price A, Gilbert N. Surveillance of Barrett's oesophagus: exploring the uncertainty through systematic review, expert workshop and economic modelling. Health Technol Assess. 2006;10(8):1-158.

3. Rubenstein J.H., Mattek N., Eisen G. Age- and sex-specific yield of Barrett's esophagus by endoscopy indication. Gastrointestinal Endoscopy. 2010;71:21-7.

4. Sharma P, Falk GW, Weston AP, Reker D, Johnston M, Sampliner RE. Dysplasia and cancer in a large multicenter cohort of patients with Barrett's esophagus. Clin Gastroenterol Hepatol. 2006;4(5):566-72.

5. Smith KJ, O'Brien SM, Smithers BM, Gotley DC, Webb PM, Green AC, et al. Interactions among smoking, obesity, and symptoms of acid reflux in Barrett's esophagus. Cancer Epidemiol Biomarkers Prev. 2005;14(11 Pt 1):2481-6.

6. Whiteman DC, Sadeghi S, Pandeya N, Smithers BM, Gotley DC, Bain CJ, et al. Combined effects of obesity, acid reflux and smoking on the risk of adenocarcinomas of the oesophagus. Gut. 2008;57(2):173-80. Epub 2007/10/13.

7. Sadeghi S, Bain CJ, Pandeya N, Webb PM, Green AC, Whiteman DC. Aspirin, non-steroidal anti-inflammatory drugs, and the risks of cancers of the esophagus. Cancer Epidemiology, Biomarkers and Prevention. 2008;17(5):1169-78.

8. Appelman HD, Umar A, Orlando RC, Sontag SJ, Nandurkar S, El-Zimaity H, et al. Barrett's esophagus: natural history. Ann N Y Acad Sci. 2011;1232:292-308. Epub 2011/09/29.

9. Wang KK, Sampliner RE. Updated guidelines 2008 for the diagnosis, surveillance and therapy of Barrett's esophagus. Am J Gastroenterol. 2008;103(3):788-97.

10. Watson A, Heading RC, Shepherd NA. Guidelines for the diagnosis and management of Barrett's columnar-lined oesophagus. Loughborough: British Society of Gastroenterology, 2005.

11. Sikkema M, de Jonge PJ, Steyerberg EW, Kuipers EJ. Risk of esophageal adenocarcinoma and mortality in patients with Barrett's esophagus: a systematic review and meta-analysis. Clin Gastroenterol Hepatol. 2010;8(3):235-44; quiz e32. Epub 2009/10/24.

12. Hameeteman W, Tytgat GN, Houthoff HJ, van den Tweel JG. Barrett's esophagus: development of dysplasia and adenocarcinoma. Gastroenterology. 1989;96(5 Pt 1):1249-56.

13. O'Connor JB, Falk GW, Richter JE. The incidence of adenocarcinoma and dysplasia in Barrett's esophagus: report on the Cleveland Clinic Barrett's Esophagus Registry. Am J Gastroenterol. 1999;94(8):2037-42.

14. Murray L, Watson P, Johnston B, Sloan J, Mainie IM, Gavin A. Risk of adenocarcinoma in Barrett's oesophagus: population based study. BMJ (Clinical Research Ed). 2003;327(7414):534-5.

15. Basu KK, Pick B, de Caestecker JS. Audit of a Barrett's epithelium surveillance database. Eur J Gastroenterol Hepatol. 2004;16(2):171-5.

16. Caygill CP, Reed PI, Hill MJ, Watson A. An initial comparison of nine centres registering patients with the UK National Barrett's Oesophagus Registry (UKBOR). Eur J Cancer Prev. 1999;8(6):539-42.

17. Shaheen N, Ransohoff DF. Gastroesophageal reflux, barrett esophagus, and esophageal cancer: scientific review. Jama. 2002;287(15):1972-81.

18. Bennett C, Vakil N, Bergman J, Harrison R, Odze R, Vieth $M$, et al. Consensus statements for management of Barrett's dysplasia and early-stage esophageal adenocarcinoma, based on a Delphi process. Gastroenterology. 2012;143(2):336-46. Epub 2012/04/28.

19. Stinnett AA, Mullahy J. Net health benefits: a new framework for the analysis of uncertainty in cost-effectiveness analysis. Med Decis Making. 1998;18(2 Suppl):S68-80.

20. Eckermann S, Briggs A, Willan AR. Health technology assessment in the cost-disutility plane. Med Decis Making. 2008;28(2):172-81. 
21. Shih YC, Halpern MT. Economic evaluations of medical care interventions for cancer patients: how, why, and what does it mean? CA Cancer J Clin. 2008;58(4):231-44.

22. Gordon LG, Eckermann S, Hirst NG, Watson DI, Mayne GC, Fahey P, et al. Healthcare resource use and medical costs for the management of oesophageal cancer. $\mathrm{Br} J$ Surg. 2011;98(11):1589-98. Epub 2011/11/08.

23. Hvid-Jensen F, Pedersen L, Drewes AM, Sorensen HT, Funch-Jensen P. Incidence of adenocarcinoma among patients with Barrett's esophagus. N Engl J Med. 2011;365(15):1375-83. Epub 2011/10/15.

24. Gordon LG, Eckermann S, Hirst NG, Watson DI, Mayne GC, Fahey P, et al. Health care resource use and medical costs for managing oesophageal cancers. British Journal of Surgery. 2011;(accepted 27/04/11).

25. Soni A, Sonnenberg A. Healthcare resource utilization in the management of oesophageal adenocarcinoma. Alimentary Pharmacology and Therapeutics. 2001;15(7):945-51.

26. Moen J, Antonov K, Larsson CA, Lindblad U, Nilsson JLG, Råstam L, et al. Factors Associated with Multiple Medication Use in Different Age Groups. Pharmacoepidemiology. 2009;43(12):197885.

27. Gordon LG, Hirst NG, Mayne GC, Watson DI, Bright T, Cai W, et al. Modeling the costeffectiveness of strategies for treating esophageal adenocarcinoma and high grade dysplasia. J Gastrointest Surg. 2012;16(8):1451-61.

28. Boger PC, Turner D, Roderick P, Patel P. A UK-based cost-utility analysis of radiofrequency ablation or oesophagectomy for the management of high-grade dysplasia in Barrett's oesophagus. Aliment Pharmacol Ther. 2010;32(11-12):1332-42.

29. Pohl H, Sonnenberg A, Strobel S, Eckardt A, Rosch T. Endoscopic versus surgical therapy for early cancer in Barrett's esophagus: a decision analysis. Gastrointest Endosc. 2009;70(4):623-31.

30. Church J, Goodall S, Gurgacz S, Whiteman DC, Lord RV. Cost-Effectiveness Of Radiofrequency Ablation Compared To Endoscopic Surveillance For Patients With Barrett's Esophagus With Low Grade Dysplasia. Value in Health. 2013;May 16(3):A215.

31. Briggs AH, Claxton K, Sculpher MJ. Decision Modelling for Health Economic Evaluation. Gray A, Briggs AH, editors. Oxford: Oxford University Press; 2006.

32. Wilson JMG, Jungner G. Principles and practice of screening for disease. Geneva: World Health Organisation, 1968.

33. Inadomi JM, Sampliner R, Lagergren J, Lieberman D, Fendrick AM, Vakil N. Screening and surveillance for Barrett esophagus in high-risk groups: a cost-utility analysis. Annals of Internal Medicine. 2003;138(3):176-86.

34. Barbiere JM, Lyratzopoulos G. Cost-effectiveness of endoscopic screening followed by surveillance for Barrett's esophagus: a review. Gastroenterology. 2009;137(6):1869-76. Epub 2009/10/21.

35. Gerson L, Lin OS. Cost-benefit analysis of capsule endoscopy compared with standard upper endoscopy for the detection of Barrett's esophagus. Clin Gastroenterol Hepatol. 2007;5(3):319-25.

36. Gerson LB, Groeneveld PW, Triadafilopoulos G. Cost-effectiveness model of endoscopic screening and surveillance in patients with gastroesophageal reflux disease. Clin Gastroenterol Hepatol. 2004;2(10):868-79.

37. Nietert PJ, Silverstein MD, Mokhashi MS, Kim CY, Glenn TF, Marsi VA, et al. Costeffectiveness of screening a population with chronic gastroesophageal reflux. Gastrointest Endosc. 2003;57(3):311-8.

38. Rubenstein JH, Inadomi JM, Brill JV, Eisen GM. Cost utility of screening for Barrett's esophagus with esophageal capsule endoscopy versus conventional upper endoscopy. Clin Gastroenterol Hepatol. 2007;5(3):312-8.

39. Benaglia T, Sharples LD, Fitzgerald RC, Lyratzopoulos G. Health benefits and cost effectiveness of endoscopic and nonendoscopic cytosponge screening for Barrett's esophagus. Gastroenterology. 2013;144(1):62-73 e6. Epub 2012/10/09. 
40. Gupta N, Bansal A, Wani SB, Gaddam S, Rastogi A, Sharma P. Endoscopy for upper GI cancer screening in the general population: a cost-utility analysis. Gastrointest Endosc. 2011;74(3):610-24 e2. Epub 2011/07/12.

41. Bhat S, Coleman HG, Yousef F, Johnston BT, McManus DT, Gavin AT, et al. Risk of malignant progression in Barrett's esophagus patients: results from a large population-based study. J Natl Cancer Inst. 2011;103(13):1049-57. Epub 2011/06/18.

42. Desai TK, Krishnan K, Samala N, Singh J, Cluley J, Perla S, et al. The incidence of oesophageal adenocarcinoma in non-dysplastic Barrett's oesophagus: a meta-analysis. Gut. 2011. Epub 2011/10/15.

43. Hirst NG, Gordon LG, Whiteman DC, Watson DI, Barendregt JJ. Is endoscopic surveillance for non-dysplastic Barrett's esophagus cost-effective? Review of economic evaluations. J Gastroenterol Hepatol. 2011;26(2):247-54. Epub 2011/01/26.

44. Das A, Wells C, Kim HJ, Fleischer DE, Crowell MD, Sharma VK. An economic analysis of endoscopic ablative therapy for management of nondysplastic Barrett's esophagus. Endoscopy. 2009;41(5):400-8.

45. Inadomi JM, Somsouk M, Madanick RD, Thomas JP, Shaheen NJ. A Cost-Utility Analysis of Ablative Therapy for Barrett's Esophagus. Gastroenterology. 2009;136(7):2101-14.

46. Provenzale D, Schmitt C, Wong JB. Barrett's esophagus: a new look at surveillance based on emerging estimates of cancer risk. Am J Gastroenterol. 1999;94(8):2043-53.

47. Sonnenberg A, Fennerty MB. Medical decision analysis of chemoprevention against esophageal adenocarcinoma. Gastroenterology. 2003;124(7):1758-66.

48. Sonnenberg A, Soni A, Sampliner RE. Medical decision analysis of endoscopic surveillance of Barrett's oesophagus to prevent oesophageal adenocarcinoma. Aliment Pharmacol Ther. 2002;16(1):41-50.

49. Husereau D, Drummond M, Petrou S, Carswell C, Moher D, Greenberg D, et al. Consolidated Health Economic Evaluation Reporting Standards (CHEERS)--explanation and elaboration: a report of the ISPOR Health Economic Evaluation Publication Guidelines Good Reporting Practices Task Force. Value in health : the journal of the International Society for Pharmacoeconomics and Outcomes Research. 2013;16(2):231-50. Epub 2013/03/30.

50. Bampton P, Schloithe A, Bull J, Padbury R, Watson D. Improving surveillance for Barrett's oesophagus. British Medical Journal. 2006;332:1320-3.

51. Amamra N, Touzet S, Colin C, Ponchon T. Current practice compared with the international guidelines: endoscopic surveillance of Barrett's esophagus. Journal of Evaluation in Clinical Practice. 2007;13(5):789-94.

52. Abrams JA, Kapel RC, Lindberg GM, Saboorian MH, Genta RM, Neugut Al, et al. Adherence to Biopsy Guidelines for Barrett's Esophagus Surveillance in the Community Setting in the United States. Clin Gastroenterol Hepatol. 2009;13:13.

53. Meining A, Ott R, Becker I, Hahn S, Muhlen J, Werner M, et al. The Munich Barrett follow up study: suspicion of Barrett's oesophagus based on either endoscopy or histology only--what is the clinical significance? Gut. 2004;53(10):1402-7.

54. Alikhan $M$, Rex D, Khan A, Rahmani E, Cummings O, Ulbright TM. Variable pathologic interpretation of columnar lined esophagus by general pathologists in community practice. Gastrointest Endosc. 1999;50(1):23-6.

55. Kim SL, Waring JP, Spechler SJ, Sampliner RE, Doos WG, Krol WF, et al. Diagnostic inconsistencies in Barrett's esophagus. Department of Veterans Affairs Gastroesophageal Reflux Study Group. Gastroenterology. 1994;107(4):945-9.

56. Galipeau PC, Li X, Blount PL, Maley CC, Sanchez CA, Odze RD, et al. NSAIDs modulate CDKN2A, TP53, and DNA content risk for progression to esophageal adenocarcinoma. PLoS Med. 2007;4(2):e67. 
57. Kountourakis P, Ajani JA, Davila M, Lee JH, Bhutani MS, Izzo JG. Barrett's Esophagus: A Review of Biology and Therapeutic Approaches. Gastrointest Cancer Res. 2012;5(2):49-57. Epub 2012/06/13.

58. Rubenstein JH, Vakil N, Inadomi JM. The cost-effectiveness of biomarkers for predicting the development of oesophageal adenocarcinoma. Aliment Pharmacol Ther. 2005;22:135-46.

59. Ramus JR, Gatenby PA, Caygill CP, Winslet MC, Watson A. Surveillance of Barrett's columnarlined oesophagus in the UK: endoscopic intervals and frequency of detection of dysplasia. Eur J Gastroenterol Hepatol. 2009;21(6):636-41.

60. Corey KE, Schmitz SM, Shaheen NJ. Does a surgical antireflux procedure decrease the incidence of esophageal adenocarcinoma in Barrett's esophagus? A meta-analysis. Am J Gastroenterol. 2003;98(11):2390-4.

61. Li YM, Li L, Yu CH, Liu YS, Xu CF. A systematic review and meta-analysis of the treatment for Barrett's esophagus. Dig Dis Sci. 2008;53(11):2837-46. Epub 2008/04/23. 